\title{
Underestimation of breast cancer in intraductal papillomas treated with vacuum-assisted core needle biopsy
}

\author{
Diana Hodorowicz-Zaniewska ${ }^{1,2}$, Benita Siarkiewicz' \\ Karolina Brzuszkiewicz ${ }^{1,2}$, Joanna Szpor ${ }^{2,3}$ \\ ${ }^{1} 7^{\text {st }}$ Chair of General Surgery, Department of General, Oncological and Gastroenterological Surgery, \\ Jagiellonian University Medical College, Cracow, Poland \\ ${ }^{2}$ University Center for Breast Diseases, University Hospital in Cracow, Poland \\ ${ }^{3}$ Chair of of Pathomorphology, Jagiellonian University Medical College, Cracow, Poland
}

\begin{abstract}
Objectives: The aims of the study were as follows: 1 ) to determine the applicability of vacuum-assisted core needle biopsy in the diagnosis and management of intraductal papillomas of the breast; 2 ) to define factors which increase the risk for underestimation of breast cancer.

Material and methods: Between 2002-2017, a total of 222 cases of intraductal papillomas were diagnosed in one center (201 using vacuum-assisted core-needle ultrasound-guided biopsy and 21 using stereotactic biopsy). All patients underwent scheduled follow-up imaging.

Results: Pure papillomas were diagnosed in 158 women, whereas papillomas with atypia, in this case atypical ductal hyperplasia (ADH), were found in 29 subjects. In the latter group, 3 cases of invasive carcinoma and 5 cases of ductal carcinoma in situ (DCIS) were detected using open surgical biopsy. Breast cancer underestimation in that group of patients was $20 \%$. Overall, $A D H$, whose presence increases the risk for BC by thirteen-fold as compared to other accompanying lesions, proved to be the most important predictive factor. Also, age, non-radical biopsy excision, and high BI-RADS ultrasound and mammogram scores increased the probability of malignancy. During the control follow-up, no cases of IP recurrence in the primary localization were observed in the group without open surgical biopsy.

Conclusions: Vacuum-assisted core needle biopsy is an efficient tool in the diagnosis and management of intraductal papillomas of the breast. Surgical excision is not indicated in cases when a pure intraductal papilloma, and data correlation between the diagnosis and the clinical presentation were confirmed. Regardless, caution is advised if residual lesions were left and in older populations. Open surgical biopsy should remain the standard of care in cases with atypia and discordance between clinical and pathology data.
\end{abstract}

Key words: intraductal papilloma; B3 breast lesions; vacuum-assisted core needle biopsy; breast carcinoma

Ginekologia Polska 2019; 90, 3: 122-127

\section{INTRODUCTION}

Intraductal papillomas (IPs) of the breast are benign growths originating from the epithelium of the milk duct. Owing to their heterogeneity and the risk for coexisting malignant growths, IPs are classified as B3, i.e. lesions of uncertain malignant potential [1]. Their incidence has been estimated at $2-3 \%$ among the female population, but the risk increases to $40-70 \%$ in case of nipple discharge [2]. Papillomas may develop in women between the ages of
30 and 77 years [3], and have either central or peripheral presentation.

Central papillomas are typically single lesions and develop in older populations. They are localized within the large collective ducts and usually manifest as serous or serosanguinous nipple discharge [4]. The risk for developing breast cancer $(\mathrm{BC})$ in women with central papillomas is comparable to the general population [5]. 
Peripheral papillomas are diagnosed in approximately $10 \%$ of the cases. They usually develop in young women and rarely manifest as nipple discharge. Peripheral papillomas present as multiple, occasionally bilateral, lesions and correlate with elevated risk for developing invasive $B C[4,6]$.

Intraductal papillomas typically present as solid, hyperechogenic growths within the milk ducts, which may result in duct enlargement, or hyperechogenic, intracystic mural nodules. In extreme cases, IPs may completely obscure the lumen of the duct or the cyst, presenting as well-differentiated, hypochogenic solid masses with smooth contours $[3,6]$. Often, IPs present as hypervascular solid masses on Doppler sonography [7]. On MMG, small IPs are usually invisible. Larger lesions present as well-differentiated, oval or round soft-tissue shadows [8], accompanied by non-suspicious microcalcifications in $25 \%$ of the cases [9]. On MRI, IPs range from normal images to irregular focal lesions, highly echogenic after the contrast agent has been administered intravenously, and exhibit the so-called wash-out effect, which is typical for malignant lesions $[8,10]$.

Histopathologic examination of core needle biopsy (CNB) specimens remains the gold standard for the diagnosis of intraductal papillomas. While the diagnosis of IPS at core needle biopsy is always associated with the need for open surgical biopsy [11-13], this is not the case for vacuum-assisted core needle biopsy (VAB). The possibility of performing the so-called therapeutic biopsy and including the affected women in the annual ultrasound control program is increasingly recommended $[14,15]$.

\section{Objectives}

The aim of the study was to determine the applicability of vacuum-assisted core needle biopsy in the management of intraductal papillomas of the breast and to identify the promoting factors for the risk of underestimation of malignant lesions.

\section{MATERIAL AND METHODS}

The study group included 222 women with IPs diagnosed using $V A B$, treated by a team of surgeons and gynecologists from a referral center for breast cancer between 2002-2017. The patients were selected from a group of women who were undergoing minimally-invasive diagnostic procedures due to changes in the breast. In total, 5113 biopsies (4083 USG-guided and 1030 MMG-guided) were performed at the time. All patients underwent an ultrasound examination of the breasts, with an additional mammogram if the subject was over 40 years of age (161 women). Between 2002-2006, VAB was performed using the Mammotome (ESS Johnson \& Johnson) system, with 11 and $8 \mathrm{G}$ needles, whereas between 2006-2017 we used the Encor and Encor Enspire (Bard) system, with 10 and 7 G needles.
Tissue samples were fixed in $10 \%$ buffered formalin, and then sent for histopathologic evaluation to the Department of Pathology.

Macroscopic evaluation of the resection margins on ultrasound or MMG was performed and logged in the medical file of each patient. In 201 cases, the diagnosis was made using ultrasound-guided biopsy, and in 21 women as a result of stereotactic biopsy.

Retrospective analysis of lesion morphology on imaging, size on MMG and USG, localization (central $\leq 3 \mathrm{~cm}$ from the nipple, and peripheral $>3 \mathrm{~cm}$ from the nipple), lesion multifocality, categorization according to the BI-RADS classification (Breast Imaging-Reporting and Data System) [16], and macroscopic evaluation of the resection margins, was performed. The following were also taken into consideration: clinical symptoms (palpable tumor, nipple discharge, mastalgia), results of physical examination, menopausal status, positive personal and family history, and hormone therapy (hormonal contraceptives or HRT for over 5 years).

Patients with IP without atypia were followed-up after 6 months, and then every 12 months. Open surgical biopsy was routinely recommended in cases with atypia or suspicious clinical-pathologic correlation (38 women).

SAS 9.4 was used for statistical analysis. Continuous variables were presented as mean and standard deviation and medians. Frequencies of the categories were calculated for discrete and ordinal variables. T-test or Mann-Whitney test were used to compare continuous variables between the groups, chi-square test and Fisher's test were used to analyze the frequencies of discrete variables. Univariate and multivariate logistic regression models were used to analyze the correlations between potential risk factors and underestimation of malignant lesions. The $p$-value of $<0.05$ was considered as statistically significant.

\section{RESULTS}

As far as all VAB are concerned, the rate of diagnosed papillomas was $4.3 \%$, which corresponded to the group of 222 women aged 22-81 years (mean: $48.2 \pm 12.8$ ). Post-menopausal, asymptomatic women, with negative personal and family history of breast cancer constituted the vast majority of these cases. Detailed clinical characteristics of the study population are presented in Table 1.

In our study, IPs were predominantly (92.3\%) single lesions, most often (60\%) in the central localization, up to $3 \mathrm{~cm}$ from the nipple. On USG, the lesions were typically well-differentiated, hypervascular solid masses, $11.5 \pm 6.2 \mathrm{~mm}$ in size (median $10 \mathrm{~mm}$ ). On MMG, the lesions were most often described as shadows or focal asymmetric densities, $16.2 \pm 12.2 \mathrm{~mm}$ in size (median $12.5 \mathrm{~mm}$ ) (Fig. 1). The vast majority of the cases were classified as BI-RADS 4a on USG, 
and only $5 \%$ presented features of highly suspicious lesions (BI-RADS 4c or 5) and (Fig. 2).

Detailed histopathologic results of all VAB are presented in Table 2.

Surgical intervention was performed in $38(17.1 \%)$ women: 22 with ADH, 4 with radial scar, 2 with DCIS, and 1 with LCIS. In 10 cases of IPs without atypia, open surgical

\begin{tabular}{|l|l|l|l|l|}
\hline \multicolumn{4}{|l|}{ Table 1. Clinical characteristics of the study population } \\
\hline & \multicolumn{3}{|l|}{ Yes } & \multicolumn{2}{l|}{ No } \\
\hline & $\begin{array}{l}\text { number } \\
{[N]}\end{array}$ & $\begin{array}{l}\text { rate } \\
{[\%]}\end{array}$ & $\begin{array}{l}\text { number } \\
{[N]}\end{array}$ & $\begin{array}{l}\text { rate } \\
{[\%]}\end{array}$ \\
\hline $\begin{array}{l}\text { Positive family history of } \\
\text { breast cancer }\end{array}$ & 62 & 27.9 & 160 & 72.1 \\
\hline $\begin{array}{l}\text { Personal history of breast } \\
\text { cancer }\end{array}$ & 3 & 1.4 & 219 & 98.6 \\
\hline Menopause & 145 & 65.3 & 77 & 34.7 \\
\hline Hormonal therapy for $>5$ years & 36 & 16.2 & 186 & 83.8 \\
\hline Clinical symptoms & 74 & 33.3 & 148 & 66.7 \\
\hline palpable tumor & 39 & 17.6 & 183 & 82.4 \\
\hline nipple discharge & 14 & 6.3 & 208 & 93.7 \\
\hline - sanguinous discharge & 16 & 7.2 & 206 & 92.8 \\
\hline - pain complaints & 14 & 6.3 & 208 & 93.7 \\
\hline - > 1 symptom & 9 & 4.1 & 213 & 95.9 \\
\hline $\begin{array}{l}\text { Peripheral localization }>3 \mathrm{~cm} \\
\text { from the nipple }\end{array}$ & 89 & 40.1 & 133 & 59.9 \\
\hline Single lesion & 192 & 86.5 & 30 & 13.5 \\
\hline Macroscopic assessment of & 205 & 92.3 & 17 & 7.6 \\
\hline
\end{tabular}

biopsy was also performed due to non-radical nature of the biopsy or lack of clinical-pathological correlation. The profile of biopsy diagnoses and histopathology results from the surgical excision is presented in Figure 3.

Notably, surgical management was abandoned in 3 cases of IPs with atypia. In 2 cases, the patients decided to discontinue that course of action, and in 1 case the decision was made because of the accompanying colon cancer spread.

In our study population, 3 (1.3\%) cases of invasive carcinoma (subtypes: ductal, lobular, and tubular) and 5 (2.2\%) cases of DCIS were found. The risk for underestimation of BC in patients with atypical ductal hyperplasia at biopsy was 20\%: $8 \%(2 / 25)$ for invasive and $12 \%$ (3/25) for non-invasive carcinoma in situ. The probability of developing invasive carcinoma in IP patients with an intraductal tumor component was $50 \%$, whereas the risk for DCIS in cases with pure papilloma was $0.6 \%(1 / 158)$.

Invasive carcinomas were diagnosed in 1 asymptomatic patient and 2 women with palpable tumors (aged 46,61 , and 66 years, respectively), and 2 subjects without positive family history. The tumors were solid (1 patient) or solid-cystic (2 patients), $12-40 \mathrm{~mm}$ in size. They were visible in both test and were classified as BIRADS $4 \mathrm{C}$ at USG and BIRADS 4 or 5 in MMG.

No correlations between the risk for $\mathrm{BC}$ underestimation and positive personal or family history, menopausal status, hormone therapy, lesion localization and patient complaints were found. However, a correlation was detected for older age ( $p=0.0015$, OR 1.7 for every 5 years) and the presence

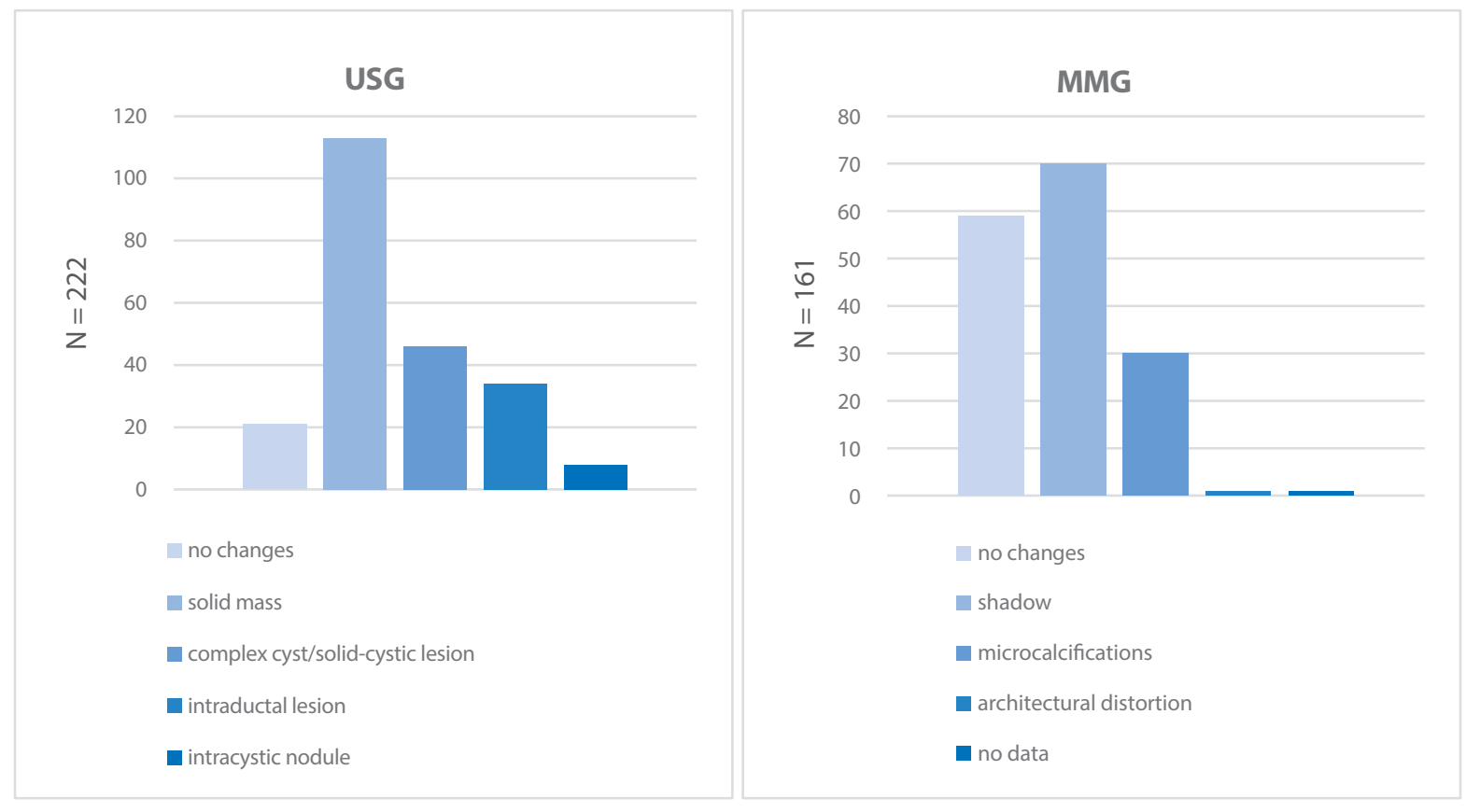

Figure 1. Morphology of the intraductal papillomas on imaging 


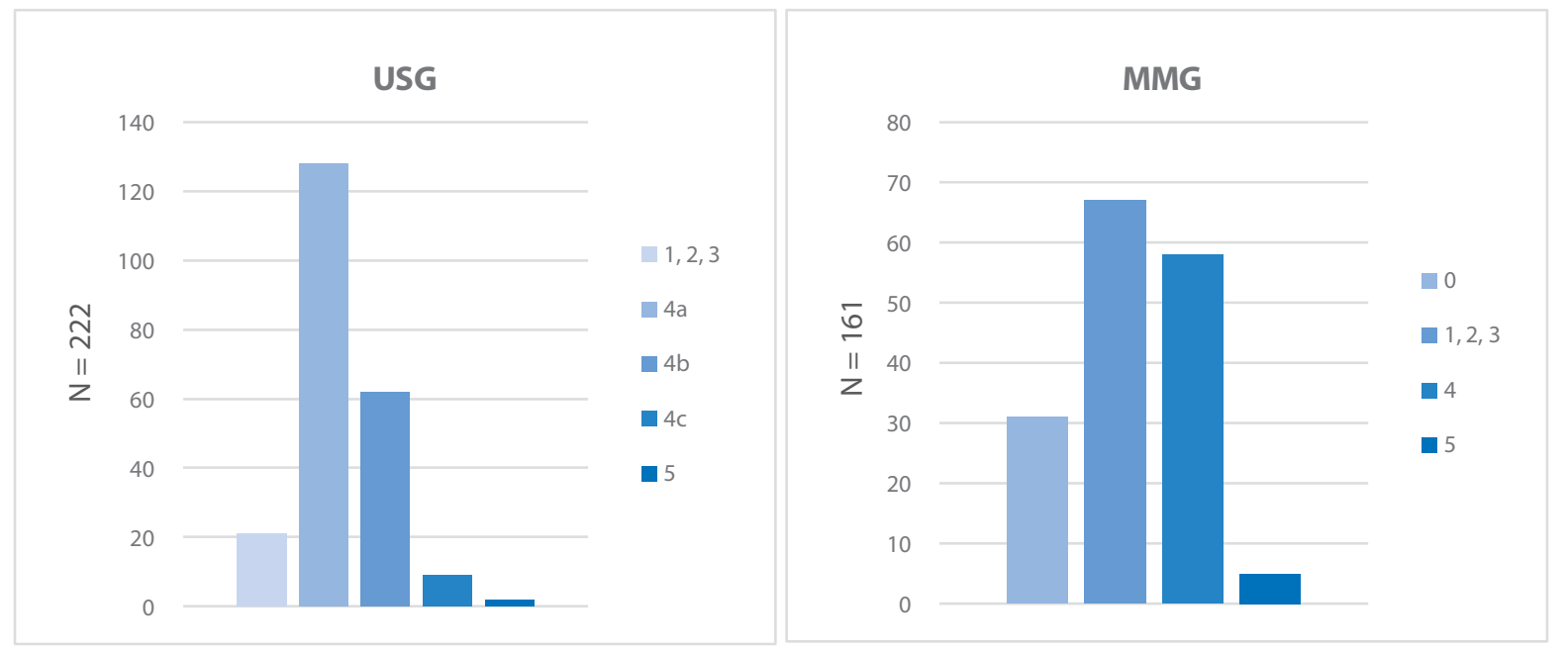

Figure 2. Categorization of the imaging results according to the BI-RADS classification [16] in the study population

\begin{tabular}{|c|c|c|}
\hline Histopathologic diagnosis & $\begin{array}{l}\text { number } \\
\text { [N] }\end{array}$ & $\begin{array}{l}\text { rate } \\
\text { [\%] }\end{array}$ \\
\hline intraductal papilloma without atypia & 158 & 70.3 \\
\hline atypical ductal hyperplasia (ADH) & 25 & 11.3 \\
\hline fibroadenoma (FA) & $20^{*}$ & 9.0 \\
\hline radial scars or complex sclerosing lesions & $17^{*}$ & 7.7 \\
\hline lobular carcinoma in situ (LCIS) & 2 & 0.9 \\
\hline ductal carcinoma in situ (DCIS) & 2 & 0.9 \\
\hline
\end{tabular}

*in 1 case intraductal papilloma was accompanied by FA and $\mathrm{ADH}$, and in 1 case by $\mathrm{ADH}$ and radial scar of atypia ( $p=0.003$, OR 16.2). The probability of BC diagnosis was also significantly higher $(p<0.001)$ in case of IP diagnosis at biopsy and BI-RADS classification (4c for USG and 4 or 5 for MMG). Identical results were obtained from the multivariate analysis (Tab. 3).

Macroscopic assessment revealed that all cases of DCIS and invasive carcinoma were detected after residual lesion biopsy. The overall risk for $\mathrm{BC}$ underestimation was $0 \%$ for the radical, and $26.7 \%$ for non-radical procedures. According to the Fisher's exact test, the $p$-value for that parameter was statistically significant $(<0.001)$.

In the group without surgical intervention, no cases of IP recurrence in the primary localization were found during the

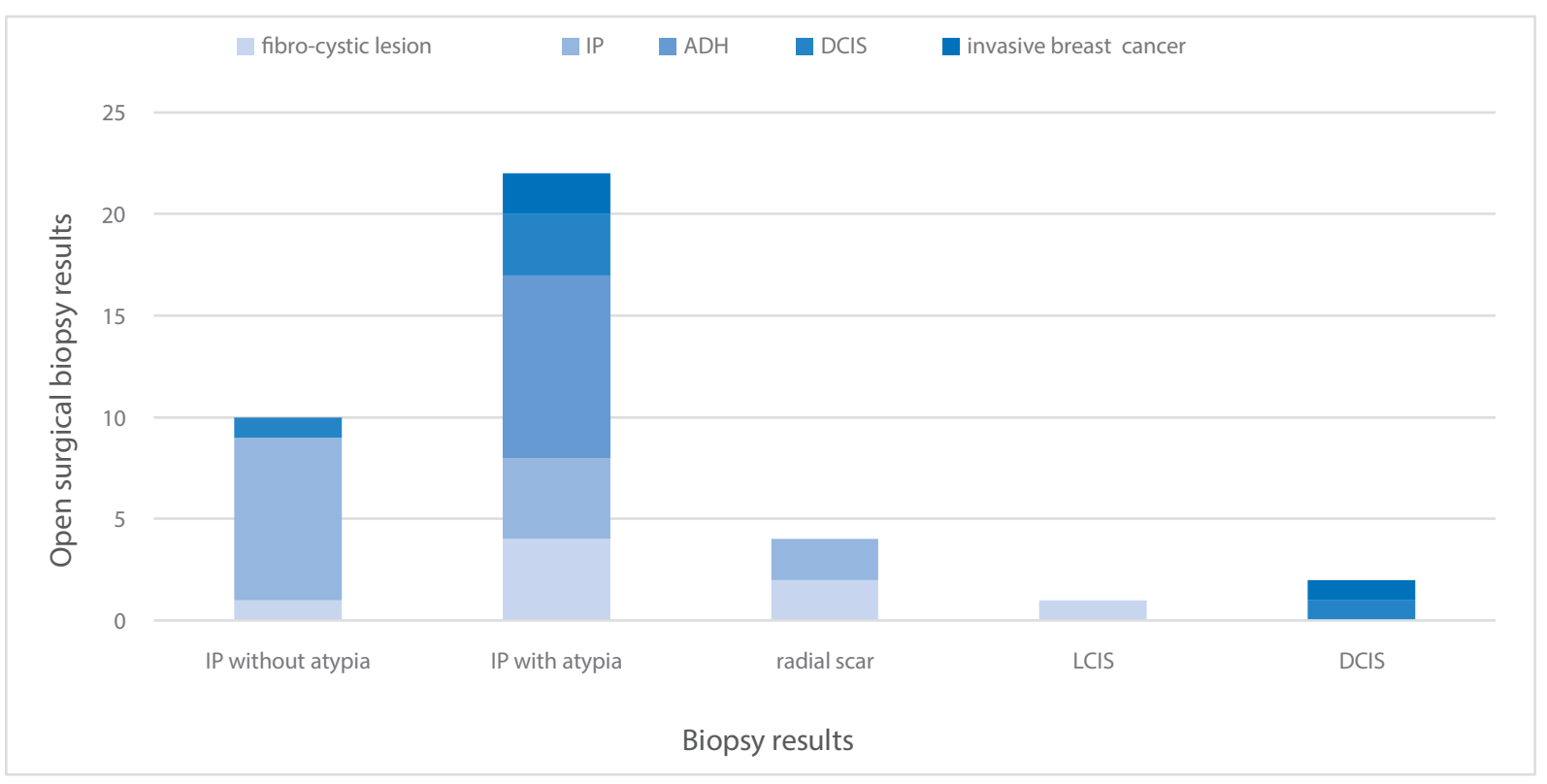

Figure 3. The profile for the final histopathologic diagnoses in the group after open surgical excision 
Table 3. Contributing factors for BC underestimation in the study population based on the multivariate analysis

\begin{tabular}{|l|l|l|}
\hline & p-value & OR \\
\hline Age & 0.029 & $1.7(1.1,2.9)$ \\
\hline BI-RADS category & 0.004 & $13.1(2.3,75.5)$ \\
\hline Atypia & 0.026 & $8.2(1.3,52.5)$ \\
\hline
\end{tabular}

follow-up control program (the control period ranged from 12 months to 16 years, mean: 28 months). New IPs in other localizations or in the other breast were found in 18 women and were again dissected using VAB. During the follow-up, 1 case of lobular carcinoma in situ, 1 of ductal carcinoma in situ, and 3 of invasive carcinomas in other localizations were diagnosed.

\section{DISCUSSION}

Intraductal papillomas belong to the heterogeneous group of lesions of uncertain malignant potential [17], which frequently presents a considerable diagnostic challenge. Due to their morphologic similarity to malignant lesions such as low-grade papillary DCIS, encapsulated papillary carcinoma, or solid papillary carcinoma, and the accompanying atypia, the diagnosis of IPs at CNB is associated with a certain risk for BC underestimation. In their 2012 meta-analysis of 34 studies from 1999-2012, Wen et al., demonstrated that the probability of IPs and an accompanying malignant process was $16.6 \%$ [18]. Therefore, until recently, open surgical biopsy was commonly performed to remove the lesions [19-21].

At present, medical advances and significant progress in the pathology-morphology diagnosis of the breasts, especially immunohistochemistry, allow for a more precise differentiation between various papillary growths [22]. Owing to that, the current underestimation of malignant $B C$ at CNB ranges from 0.4 to $4 \%[23,24]$. Also, due to the development of minimally invasive techniques, e.g.VAB, the standards of management have changed and the indications for surgical interventions have been distinctly limited. The method in question allows to obtain an almost unlimited number of large specimens from one puncture site, and in case of pure papillomas it offers an equal in quality therapeutic option. As far as IPs with atypia are concerned, the risk for underestimation at CNB remains considerable, from 13 to $92 \%$ according to the available literature [25]. In light of the fact that VAB does not allow for a histological evaluation of the resection margins, open surgical biopsy is routinely recommended [14, 26, 27].

The risk for underestimation of a malignant process is notably smaller in case of vacuum-assisted CNB as compared to standard CNB, and has been estimated at $0 \%-2.6 \%[14,28]$ versus 9-21\% [15, 29] for pure IPs versus IPs with atypia [15, 29].
Our findings are consistent with the literature. The risk for $\mathrm{BC}$ underestimation was $0.6 \%$ at VAB. The accompanying atypical ductal hyperplasia, as compared to other histological diagnoses, increased the risk for malignancy by 13 -fold, reaching $20 \%$.

The role of atypical growths as the most important predictive factors for a malignant process was also emphasized in the meta-analysis by Wen [21], or later works by Shiino, Han, Kiran, Boufelli or Foley [12, 23-25, 30]. Current recommendations on diagnostic-therapeutic management still advise radical surgical approach in IPs with atypia, regardless of the biopsy technique [31]. Conservative management is allowed in strictly selected groups of patients [14, 27].

In our study, we detected an increased risk for underestimation in older populations, which is consistent with the reports by Foley et al., Rasmussen et al., and Yu et al. $[30,32,33]$. Therefore, caution is advised when recommending conservative management to that group of patients. On the other hand, we did not prove the predictive meaning of the accompanying clinical symptoms such as sanguine nipple discharge or multifocality of the lesions, which has been emphasized by Han et al. [23]. Also, similarly to Rasmussen, we found no correlation between the volume of the lesions at imaging and the risk for $\mathrm{BC}$ underestimation, which has been suggested by Boufelli et al., and Yu et al. $[25,33]$. We believe it is not the volume of the lesion but the evaluation of sample representativeness and the results of clinical-pathological correlation which play the crucial role in the process. In our study, insufficient resection margins confirmed at follow-up control imaging and benign result of VAB in patients with high BI-RADS classification/scores, USG (at least 4c) and MMG (4 or 5) notably increased the risk for cancer underestimation. Similar correlations were presented by Wen et al., and Yu et al., for IPs and by Williams et al., for atypia [18, 33, 34].

We were not able to identify morphological features of the lesions on imaging which might become statistically significant predictors of benign or malignant nature of the change, which is consistent with the reports of Rasmussen et al. [32].

As no IP recurrence was observed during the follow-up period, it seems safe to assume that VAB is an effective method of IPs removal. However, due to the visible tendency for the development of new lesions in other localizations and increased BC incidence [13], we are of the opinion that the affected women should remain in the control follow-up program.

\section{CONCLUSIONS}

Vacuum-assisted core needle biopsy is an effective technique in the diagnosis and management of intraductal papillomas. Our findings, as well as the reports of other 
authors, indicate that the diagnosis of pure papilloma and corresponding clinical presentation do not require further surgical intervention. That said, caution and careful monitoring are advised in older populations and cases with residual lesions. Radical surgical biopsy should remain the method of choice for all cases of IPs with atypia and lack of clinical-pathological correlation.

\section{REFERENCES:}

1. Ellis IO. Best Practice No 179: Guidelines for breast needle core biopsy handling and reporting in breast screening assessment. Journal of Clinical Pathology. 2004; 57(9): 897-902, doi: 10.1136/jcp.2003.010983.

2. Dietz JR, Crowe JP, Grundfest $S$, et al. Directed duct excision by using mammary ductoscopy in patients with pathologic nipple discharge. Surgery. 2002; 132(4): 582-7; discussion 587, indexed in Pubmed: 12407341.

3. Ganesan S, Karthik G, Joshi M, et al. Ultrasound spectrum in intraductal papillary neoplasms of breast. Br J Radiol. 2006; 79(946): 843-849, doi: 10.1259/bjr/69395941, indexed in Pubmed: 16641415.

4. Al Sarakbi W, Worku D, Escobar PF, et al. Breast papillomas: current management with a focus on a new diagnostic and therapeutic modality. Int Semin Surg Oncol. 2006; 3: 1-8, doi: 10.1186/1477-7800-3-1, indexed in Pubmed: 16417642

5. Haagensen $C D$, Bodain $C$, Haagensen DE. Breast carcinoma risk and detection. WB Saunders, Philadelphia 1981: 146.

6. Oyama T, Koerner FC. Noninvasive papillary proliferations. Semin Diagn Pathol. 2004; 21(1): 32-41, indexed in Pubmed: 15074557.

7. Brookes MJ, Bourke AG. Radiological appearances of papillary breast lesions. Clin Radiol. 2008; 63(11): 1265-1273, doi: 10.1016/j. crad.2008.02.012, indexed in Pubmed: 18929044.

8. Eiada R, Chong J, Kulkarni S, et al. Papillary lesions of the breast: MRI, ultrasound, and mammographic appearances. AJR Am J Roentgenol. 2012; 198(2): 264-271, doi: 10.2214/AJR.11.7922, indexed in Pubmed: 22268167.

9. Cardenosa G, Eklund GW. Benign papillary neoplasms of the breast: mammographic findings. Radiology. 1991; 181(3): 751-755, doi: 10.1148/radiology.181.3.1947092, indexed in Pubmed: 1947092.

10. Daniel BL, Gardner RW, Birdwell RL, et al. Magnetic resonance imaging of intraductal papilloma of the breast. Magn Reson Imaging. 2003; 21 (8): 887-892, indexed in Pubmed: 14599539.

11. Rasmussen BB, Balslev E, Jarle Christensen lb, et al. Diagnostic challenges in clinical, radiological and histopathological tests regarding papillomatous lesions of the breast. Breast. 2018; 40: 177-180, doi: 10.1016/j. breast.2018.05.006, indexed in Pubmed: 29906740.

12. Shiino $\mathrm{S}$, Tsuda $\mathrm{H}$, Yoshida $\mathrm{M}$, et al. Intraductal papillomas on core biopsy can be upgraded to malignancy on subsequent excisional biopsy regardless of the presence of atypical features. Pathol Int. 2015; 65(6): 293-300, doi: 10.1111/pin.12285, indexed in Pubmed: 25801805.

13. Khan S, Diaz A, Archer KJ, et al. Papillary lesions of the breast:To excise or observe? Breast J. 2018; 24(3): 350-355, doi: 10.1111/tbj.12907, indexed in Pubmed: 28845569.

14. Rageth CJ, O'Flynn EAm, Comstock C, et al. First International Consensus Conference on lesions of uncertain malignant potential in the breast (B3 lesions). Breast Cancer Res Treat. 2016; 159(2): 203-213, doi: 10.1007/s10549-016-3935-4, indexed in Pubmed: 27522516.

15. Saladin C, Haueisen H, Kampmann G, et al. MIBB Group. Lesions with unclear malignant potential (B3) after minimally invasive breast biopsy: evaluation of vacuum biopsies performed in Switzerland and recommended further management. Acta Radiol. 2016; 57(7): 815-821, doi: 10.1177/0284185115610931, indexed in Pubmed: 26552694.

16. D'Orsi CJ, Sickles EA, Mendelson EB. et al.. ACR BI-RADS Atlas: Breast Imaging Reporting and Data System. 5th ed. American College of Radiology, Reston 2013.
17. Hoda S, Kaplan R. World Health Organization (WHO) Classification of Breast Tumours, 4th ed. The American Journal of Surgical Pathology. 2013; 37(2): 309-310, doi: 10.1097/pas.0b013e318273b19b.

18. Wen $X$, Cheng W. Nonmalignant breast papillary lesions at core-needle biopsy: a meta-analysis of underestimation and influencing factors. Ann Surg Oncol. 2013; 20(1): 94-101, doi: 10.1245/s10434-012-2590-1, indexed in Pubmed: 22878621.

19. Collins LC, Schnitt SJ. Papillary lesions of the breast: selected diagnostic and management issues. Histopathology. 2008; 52(1): 20-29, doi: 10.1111/j.1365-2559.2007.02898.x, indexed in Pubmed: 18171414.

20. Jacobs TW, Connolly JL, Schnitt SJ. Nonmalignant lesions in breast core needle biopsies: to excise or not to excise? Am J Surg Pathol. 2002; 26(9): 1095-1110, indexed in Pubmed: 12218567.

21. Shiino S, Tsuda $H$, Yoshida M, et al. Intraductal papillomas on core biopsy can be upgraded to malignancy on subsequent excisional biopsy regardless of the presence of atypical features. Pathol Int. 2015; 65(6): 293-300, doi: 10.1111/pin.12285, indexed in Pubmed: 25801805.

22. Agoumi M, Giambattista J, Hayes MM. Practical Considerations in Breast Papillary Lesions: A Review of the Literature. Arch Pathol Lab Med. 2016; 140(8): 770-790, doi: 10.5858/arpa.2015-0525-RA, indexed in Pubmed: 27472236.

23. Han SH, Kim M, Chung YRi, et al. Benign Intraductal Papilloma without Atypia on Core Needle Biopsy Has a Low Rate of Upgrading to Malignancy after Excision. J Breast Cancer. 2018; 21(1): 80-86, doi: 10.4048/jbc.2018.21.1.80, indexed in Pubmed: 29628987.

24. Kiran S, Jeong YJu, Nelson ME, et al. Are we overtreating intraductal papillomas? J Surg Res. 2018; 231: 387-394, doi: 10.1016/j.jss.2018.06.008, indexed in Pubmed: 30278958.

25. Boufelli G, Giannotti MA, Ruiz CA, et al. Papillomas of the breast: factors associated with underestimation. Eur J Cancer Prev. 2018; 27(4): 310-314, doi: 10.1097/CEJ.0000000000000343, indexed in Pubmed: 28296665.

26. Am Soc Breast Surg. Consensus guideline on concordance assessment of image-guided breast biopsies and management of borderline or high-risk lesions. 2016, Nov 2 . https://www.breastsurgeons. org/about/statements/PDF_Statements/Concordance_and_High\%20 RiskLesions.pdf.

27. Nowecki Zl, Jeziorski A, eds . Chirurgiczne leczenie zmian nowotworowych piersi. In: (eds). Biblioteka chirurga onkologa..Vol 5. Via Medica, Gdańsk 2016: 125.

28. Mosier AD, Keylock J, Smith DV. Benign papillomas diagnosed on large-gauge vacuum-assisted core needle biopsy which span $<1.5 \mathrm{~cm}$ do not need surgical excision. Breast J. 2013; 19(6): 611-617, doi: 10.1111/tbj.12180, indexed in Pubmed: 24102818.

29. Chang JM, Han W, Moon WK, et al. Papillary lesions initially diagnosed at ultrasound-guided vacuum-assisted breast biopsy: rate of malignancy based on subsequent surgical excision. Ann Surg Oncol. 2011; 18(9): 25062514, doi: 10.1245/s10434-011-1617-3, indexed in Pubmed: 21369740.

30. Foley NM, Racz JM, Al-Hilli Z, et al. An International Multicenter Review of the Malignancy Rate of Excised Papillomatous Breast Lesions. Ann Surg Oncol. 2015; 22 Suppl 3: S385-S390, doi: 10.1245/s10434-015-4773-Z, indexed in Pubmed: 26240010.

31. Rageth CJ, O'Flynn EAM, Pinker K, et al. Second International Consensus Conference on lesions of uncertain malignant potential in the breast (B3 lesions). Breast Cancer Res Treat. 2018 [Epub ahead of print], doi: 10.1007/s10549-018-05071-1, indexed in Pubmed: 30506111.

32. Rasmussen BB, Balslev E, Jarle Christensen Ib, et al. Diagnostic challenges in clinical, radiological and histopathological tests regarding papillomatous lesions of the breast. Breast. 2018; 40: 177-180, doi: 10.1016/j. breast.2018.05.006, indexed in Pubmed: 29906740.

33. Yu Y, Salisbury E, Gordon-Thomson D, et al. Management of papillary lesions without atypia of the breast diagnosed on needle biopsy. ANZ J Surg. 2018 [Epub ahead of print], doi: 10.1111/ans.14827, indexed in Pubmed: 30414221.

34. Williams KE, Amin A, Hill J, et al. Radiologic and Pathologic Features Associated With Upgrade of Atypical Ductal Hyperplasia at Surgical Excision. Acad Radiol. 2018 [Epub ahead of print], doi: 10.1016/j.acra.2018.09.010, indexed in Pubmed: 30318287. 